\title{
A Systematic Procedure to Study the Influence of Occupant Behavior on Building Energy Consumption
}

\author{
Zhun $\mathrm{Yu}^{1}$, Benjamin C. M. Fung ${ }^{2}$, Fariborz Haghighat ${ }^{1}$, Hiroshi Yoshino ${ }^{3}$, Edward \\ Morofsky 4 \\ ${ }^{1}$ Department of Building, Civil and Environmental Engineering, Concordia University, \\ Montreal, Quebec, H3G 1M8, Canada \\ ${ }^{2}$ Concordia Institute for Information Systems Engineering, Concordia University, \\ Montreal, Quebec, H3G 1M8, Canada \\ ${ }^{3}$ Department of Architecture and Building Science, Tohoku University, Japan \\ ${ }^{4}$ Real Property Branch, Public Works and Government Services Canada, Place du Portage III, \\ 8B1, Gatineau, Québec, K1A 0S5 Canada
}

\begin{abstract}
Efforts have been devoted to the identification of the impacts of occupant behavior on building energy consumption. Various factors influence building energy consumption at the same time, leading to the lack of precision when identifying the individual effects of occupant behavior. This paper reports the development of a new methodology for examining the influences of occupant behavior on building energy consumption; the method is based on a basic data mining technique (cluster analysis). To deal with data inconsistencies, min-max normalization is performed as a data preprocessing step before clustering. Grey relational grades, a measure of relevancy between two factors, are used as weighted coefficients of different attributes in cluster analysis. To demonstrate the applicability of the proposed method, the method was applied to a set of residential buildings' measurement data. The results show that the method facilitates the evaluation of building energy-saving potential by improving the behavior of building occupants, and provides multifaceted insights into building energy end-use patterns associated with the occupant behavior. The results obtained could help prioritize efforts at modification of occupant behavior in order to reduce building energy consumption, and help improve modeling of occupant behavior in numerical simulation.
\end{abstract}

Keywords: Occupant behavior; Building energy consumption; Data mining; Cluster analysis; Grey relational analysis 


\section{Introduction}

The identification of major determinants of building energy consumption, together with a thorough understanding of the impacts of the identified determinants on energy consumption patterns, could assist in achieving the goal of improving building energy performance and reducing greenhouse gas emissions due to the building energy consumption. In general, the factor influencing the total building energy consumption can be divided into seven categories:

(1) Climate (e.g., outdoor air temperature, solar radiation, wind velocity, etc.),

(2) Building-related characteristics (e.g., type, area, orientation, etc.)

(3) User-related characteristics, except for social and economic factors (e.g., user presence, etc.),

(4) Building services systems and operation (e.g., space cooling/heating, hot water supplying, etc.),

(5) Building occupants' behavior and activities,

(6) Social and economic factors (e.g., degree of education, energy cost, etc.), and

(7) Indoor environmental quality required.

Among these seven factors, social and economic factors will partly determine the occupant attitude toward energy consumption, and building occupants will embody such impact on their daily activities and behavior, thereby influencing building energy consumption. At the same time, indoor environment quality could be regarded as being basically decided by building occupants, thereby influencing building energy consumption. In essence, these two categories of factors which represent occupants' influences affect building energy consumption indirectly. Therefore, their influences on building energy consumption are already contained within the effects of occupant behavior, and there is no need to take them into consideration when identifying the effects of influencing factors.

The separate and combined influences of the first four factors on building energy consumption can be identified via simulation. With a variety of parameter settings, current simulation software is robust in respect to simulating different situations based upon these four factors. However, it is difficult to completely identify the influences of occupant behavior and activities through simulation due to users' behavior diversity and complexity; current simulation tools can only imitate behavior patterns in a rigid way. In recent years several models have been established to integrate the influence of building occupant behavior into building simulation programs [1-4]. However, these models focus only on typical activities such as the control of sun-shading devices, while realistic building user-behavior patterns are more complicated.

A number of studies [5-7] suggest that, in order to obtain the full effects of user behavior, one possible approach is to extract corresponding useful information from real measured data, since such data already contains the full effects. For example, Yu et al [7] proposed a decision tree method for building energy demand modeling, and applied this method to the historical data on Japanese residential buildings. The 
generated model has a flowchart-like tree structure, enabling users to quickly extract useful information on the influence factors of building energy consumption. Such model along with derived information could benefit the improvement of building energy performance greatly. Generally, the previous studies on the effects of occupant behavior can be divided into two categories. The first category focuses on the effects of building user presence on building energy consumption. For example, Emery and Kippenhan [8] reported a survey on the effects of occupant presence upon home energy usage in four nearly identical houses. The four houses were divided into two pairs, and the building envelope of one pair was constructed with improved thermal resistance. One of each pair of houses was left unoccupied, while the other was occupied by university student families. Researchers compared the first heating season's (1987-88) total energy consumption of the occupied and unoccupied houses (i.e., the sum of heating, lighting, and appliances). They found that the presence of occupants increased the total energy consumption of both occupied houses, and the house with the improved building envelope had a smaller increase. The second category focuses on the effects of actions occupants took to influence energy consumption. For example, Ouyang and Hokao [9] investigated energy-saving potential by improving user behavior in 124 households in China. In this study, these houses were divided into two groups: one was educated to promote energy-conscious behavior and put corresponding energy-saving measures into effect in July 2008, while the other was required to keep behavior intact. Comparisons were made between monthly household electricity uses in July 2007 and July 2008 for both groups. Researchers found that, on the average, effective promotion of energy-conscious behavior could reduce household electricity consumption by more than $10 \%$. Evidently, comparative analyses on measured data were conducted in these studies to identify the effects of user behavior. However, the limitations of this method are significant. First, apart from user behavior, the other four influencing factors also contribute to the variation in building energy consumption simultaneously, while this method is unable to adequately remove the effects of those four factors and identify the influences of occupant behavior. Although in these studies some measures were implemented to remove the impact of those factors, such as using nearly identical housing characteristics and taking energy data in other years with similar climatic conditions as a reference, the effects of these measures are questionable since even a slight difference in some building parameters (e.g., heat loss coefficient) and weather parameters (e.g., annual average outdoor air temperature) would result in remarkable fluctuations in the building energy consumption. Second, in real building databases, buildings are usually described by a mixture of variable types such as numerical variable, categorical variable (e.g., residential building types are divided into detached and apartment), and ordinal variable (e.g., buildings are rated as platinum, gold, and silver). Such data of mixed variable types is difficult to process by statistical methods that are normally utilized in comparative analyses. This also adds the difficulty of distinguishing between building-related effects and user-related effects. Third, with regard to comparative analyses, buildings are usually classified into different groups to simplify research. Such classification is commonly based on building-related 
parameters, such as floor area. For example, if building floor area ranges from $100 \mathrm{~m}^{2}$ to $400 \mathrm{~m}^{2}$, it can be replaced by small, medium, and large corresponding to the intervals [100, 200], [200, 300], and [300, 400], respectively. Accordingly, all the buildings are classified into three groups, i.e. small buildings, medium buildings, and large buildings; and further study can be performed on each group. In this process, the partition of building-related parameters is normally decided by considerations of convenience and intuition. Why should $200 \mathrm{~m}^{2}$ and $300 \mathrm{~m}^{2}$ be the interval between each group? Hence, a more rational classification method for grouping buildings is required.

Moreover, buildings are commonly represented by various typical parameters at the same time, such as building age and floor area. All these parameters may be divided into different levels, such as low and high, for simplicity. In order to perform a comprehensive investigation, the sample size (i.e. number of buildings) necessary for research should be determined by the combination of different levels of all parameters. For example, suppose seven typical parameters are selected for representation and each are stratified into 3 levels (e.g. small, medium, and large). In terms of combinatorial theory, it can be calculated that at least $3^{7}=2187$ buildings should be investigated for comparison, which may be quite impractical.

The main purpose of this paper is to develop a methodology for identifying the effects of occupant behavior on the building energy consumption through data analysis, thereby evaluating the energy saving potential by improving user behavior and providing deep insights into the building energy consumption patterns.

This paper is organized as follows: Section 2 introduces the proposed methodology. Section 3 describes the results of applying this method to a set of field measurement data and discusses the related work. Section 4 concludes the paper.

\section{Methodology}

A new methodology is proposed for examining the effects of occupant behavior on the building energy consumption. Basically, it is realized by organizing similar buildings among all the investigated buildings into various groups based on the four influencing factors unrelated to user behavior, so that for each building in the same group the four factors have similar effects on the building energy consumption. Accordingly, the effects of occupant behavior on the building energy consumption can be identified accurately in these groups. Further, provided there is a sufficient building sample size and subject buildings have a large divergence in the four influencing factors, implying that the full effects of the four factors in each group can be similar enough and the energy consumption difference caused by them is comparatively small, energy consumption difference between buildings in each group could be thought of as being caused only by occupant behavior. It is obvious that the identification of building groups is the most important element of this methodology. Such identification is 
achieved mainly via cluster analysis.

\subsection{Cluster analysis}

Cluster analysis is the process of grouping the observations into classes or clusters so that objects in the same cluster have high similarity, while objects in different clusters have low similarity. Fig. 1 shows a clustering schema based on a hypothetical building data table. It contains various energy-related variables such as outdoor air temperature (T) and building heat loss coefficient (HLC).

\begin{tabular}{|c|c|c|c|c|}
\hline \multirow{4}{*}{ Cluster 1} & Instance & $\begin{array}{l}\text { Attribute } 1 \\
\text { (T) }\end{array}$ & $\ldots$ & $\begin{array}{l}\text { Attribute m } \\
\text { (HLC) }\end{array}$ \\
\hline & Instance 1 & $\mathrm{x}$ & $\mathrm{x}$ & $\mathrm{x}$ \\
\hline & $\ldots$ & $\mathrm{x}$ & $\mathrm{X}$ & $\mathrm{x}$ \\
\hline & Instance $\mathrm{i}$ & $\mathrm{x}$ & $\mathrm{x}$ & $\mathrm{X}$ \\
\hline \multirow{2}{*}{$:$} & $\ldots$ & $\mathrm{x}$ & $\mathrm{x}$ & $\mathrm{x}$ \\
\hline & Instance $\mathrm{j}$ & $\mathrm{x}$ & $\mathrm{X}$ & $\mathrm{x}$ \\
\hline \multirow[t]{2}{*}{ Cluster w } & $\ldots$ & $\mathrm{x}$ & $\mathrm{x}$ & $\mathrm{x}$ \\
\hline & Instance $n$ & $\mathrm{X}$ & $\mathrm{X}$ & $\mathrm{X}$ \\
\hline
\end{tabular}

Figure 1. Clustering schema

The data table consists of $m$ attributes and $n$ instances. Each attribute represents a variable and each instance denotes a building. All the instances are grouped into $w$ clusters. Accordingly, these $w$ clusters are homogeneous internally and heterogeneous between different clusters [10]. Such internal cohesion and external separation are based upon the $m$ attributes as well as their influences; it implies that these attributes have the most similar holistic effects on the building energy performance of the same cluster buildings, while the effects are significantly distinct for the buildings in different clusters. Therefore, the separate effects of occupant behavior on the building energy consumption can be identified more precisely based on cluster analysis and the four influencing factors unrelated to the occupant behavior. Note that these four influencing factors are represented by corresponding parameters selected from an existing database.

Before conducting cluster analysis, some preprocessing steps are needed in order to deal with the inconsistencies of different attributes. For example, most of the energy-related attributes have their own units. Switching attribute units from one to another may significantly change the attribute values, thereby impacting the quality and accuracy of clusters. Therefore, data transformation techniques should be applied in order to help avoid dependence on the selection of attribute units. Also, data transformation can help prevent attributes with large ranges from outweighing those with comparatively smaller ranges. At the same time, the contribution of different attributes to the building energy consumption may differ considerably; thus, after data normalization, each attribute should be associated with a weight that reflects its significance. Grey relational analysis will be used to identify such weights. The procedure of data transformation and grey relational analysis will be introduced in Sections 2.2 and 2.3 , respectively. 
The dissimilarity between observations in the database is calculated using the distance between them in the cluster analysis. In this study, the most popular distance measure, Euclidean distance, is used [10]:

$$
d(k, l)=\sqrt{\left(x_{k 1}-x_{l 1}\right)^{2}+\left(x_{k 2}-x_{l 2}\right)^{2}+\cdots+\left(x_{k n}-x_{l n}\right)^{2}}
$$

where $k=\left(x_{k 1}, x_{k 2}, \ldots, x_{k n}\right)$ and $l=\left(x_{l 1}, x_{l 2}, \ldots, x_{l n}\right)$ are buildings. $x_{k 1}, \ldots, x_{k n}$ are $n$ parameters of $k$ and $x_{l 1}, \ldots, x_{l n}$ are $n$ parameters of $l$.

Commonly used clustering algorithms include K-means, K-medoids, and CLARANS [10]. In this study, we employ the K-means, along with open-source data mining software WEKA [11], to perform cluster analysis, due to its high efficiency and wide applicability.

The K-means algorithm is one of the simplest partition methods to solve clustering problem. Given a dataset $(D)$ containing $w$ objects, the K-means algorithm aims to partition these $w$ objects into $k$ clusters with two restraints: 1) the center of each cluster is the mean position of all objects in that cluster, 2) each object has been assigned to the cluster with the closest center. This algorithm consists of given steps: 1) Randomly select $k$ observations from $D$ as the initial cluster centers, 2) Calculate the distance between each remaining observation and each initially chosen center, 3) Assign each remaining observation to the cluster with the closest center, 4) Recalculate the mean values, i.e., the cluster centers, of the new clusters, and 5) Repeat Steps 2 to 4 until the algorithm converges, meaning that the cluster centers do not change. It should be mentioned that $\mathrm{K}$-means is quite sensitive to initial cluster centers. Therefore, different values should be tried so as to obtain the minimum sum of the distances within a cluster. At the same time, the number of clusters should be specified in advance.

\subsection{Data transformation}

As mentioned previously, data transformation has been applied in order to deal with the inconsistencies in measured dataset. Specifically, min-max normalization [10] is performed to scale the values so that they fall within a predetermined range. The main advantage of min-max normalization lies in its ability to reserve the relationships between the initial data since it carries out a linear normalization. Assume that $x_{\max }$ and $x_{\min }$ are the original maximum and minimum values of a numerical attribute. By min-max normalization, a value, $x$, of this attribute can be transformed to $x$ ' in the new specified range $\left[x_{\text {min }}^{\prime}, x_{\text {max }}^{\prime}\right]$ by calculating

$$
x^{\prime}=\frac{x-x_{\text {min }}}{x_{\text {max }}-x_{\text {min }}}\left(x_{\text {max }}^{\prime}-x_{\text {min }}^{\prime}\right)+x_{\text {min }}^{\prime}
$$

In this study, the new range is defined as $[0,1]$.

For binary attributes, their two states, such as the operation states of room air conditioners, i.e. $[\mathrm{ON}, \mathrm{OFF}]$, can be transformed to $[0,1]$ or $[1,0]$ directly. The decision to recode these two states to either $[0,1]$ or $[1,0]$ depends upon whether or not there is a preferred positive value. 
For multi-valued categorical attributes with an implicit order, it is often necessary to rank their ordered states first, and then map the obtained range onto $[0,1]$ by

$$
x_{i}^{\prime}=\frac{\operatorname{rank}_{i}-1}{\operatorname{rank}_{\max }-1}
$$

where

$x$ ': transformed value of each state

rank $_{i}$ : corresponding rank of each state

rank $_{\text {max }}$ : maximum rank

For example, the four levels of certification in the Leadership in Energy and Environmental Design (LEED) Green Building Rating System, i.e. [CERTIFIED, SILVER, GOLD, PLATINUM], will be transformed to $[0,1 / 3,2 / 3,1]$ using the aforementioned method.

\subsection{Grey relational analysis}

Based on geometrical mathematics, grey relational analysis (GRA) has been proposed in order to find grey relational grades and a grey relational order (i.e. the rank of grey relational grades) that can be used to describe primary trend relationships between related factors, and to identify the important factors that significantly influence predefined target factors [12]. For example, if the building energy consumption is defined as the target factor, GRA can provide grey relational grades for its various influencing factors, such as outdoor air temperature and floor area. These grey relational grades are numerical measures of the impact of the influencing factors on the total building energy consumption. The larger the grey relational grades are, the more significant impacts the influencing factors have. In comparison with other similar multi-factorial analysis methods such as regression analysis and principal component analysis, the main advantages of GRA are its comparative simplicity and the ability to deal with small data sets that do not have typical probability distributions.

Let $y_{0}$ be the objective sequence (measured data of target factor, such as the building energy consumption) and $y_{i}$ be the compared sequences (measured data of related factors, such as various influencing factors of building energy consumption):

The procedure of GRA is described as follows:

$$
\begin{aligned}
& y_{0}=\left(y_{0}(1), \mathrm{y}_{0}(2), \ldots, \mathrm{y}_{0}(n)\right) \\
& \mathrm{y}_{i}=\left(\mathrm{y}_{i}(1), \mathrm{y}_{i}(2), \ldots, \mathrm{y}_{i}(n)\right), \quad i=1,2, \ldots, m
\end{aligned}
$$

Step 1: Normalization of raw data (Min-max normalization is used in this study), $y_{0}$ and $y_{i}$ are used to denote obtained normalized sequences;

Step 2: Calculate grey relational coefficients $\zeta . \zeta_{i}(k)$ between $y_{0}$ and $y_{i}$ is defined as

$$
\begin{gathered}
\xi_{i}(k)=\frac{\min _{i} \min _{k}\left|y_{0}(k)-y_{i}(k)\right|+\alpha \max _{i} \max _{k}\left|y_{0}(k)-y_{i}(k)\right|}{\left|y_{0}(k)-y_{i}(k)\right|+\alpha \max _{i} \max _{k}\left|y_{0}(k)-y_{i}(k)\right|} \\
i=1,2, \ldots, \mathrm{m} ; k=1,2, \ldots, \mathrm{n}
\end{gathered}
$$

where $\alpha$ is distinguishing coefficient and $0<\alpha<1$, normally $\alpha=0.5$;

Step 3: Calculate grey relational grade $\gamma$ 


$$
\gamma\left(y_{0}, y_{i}\right)=\frac{1}{n} \sum_{k=1}^{n} \xi_{i}(k)
$$

Step 4: Rank the obtained grey relational grades; thus, grey relational order can be identified.

As mentioned previously, grey relational grade will be employed to be weighted coefficients of corresponding attributes in cluster analysis. Note that grey relational grades range from 0 to 1 . Generally, $r>0.9$ indicates a marked influence, $r>0.8$ indicates a relatively marked influence, $r>0.7$ indicates a noticeable influence, and $r$ $<0.6$ indicates a negligible influence [13].

\section{Case study - Occupant behavior effects in residential buildings}

\subsection{Data collection and preprocessing}

To evaluate and improve residential buildings' energy performance, a project entitled "Investigation on Energy Consumption of Residents All over Japan" was carried out by the Architecture Institute of Japan from December 2002 to November 2004 [14]. For this project, field surveys on energy-related data and other relevant information were carried out in 80 residential buildings located in six different districts in Japan: Hokkaido, Tohoku, Hokuriku, Kanto, Kansai, and Kyushu. Table 1 shows the survey items and corresponding investigation methods. Fig. 2 shows measuring instruments which were used to monitor temperature and consumptions of electricity, gas, and/or kerosene.

Table 1: Investigation items and methods

\begin{tabular}{|c|c|c|c|}
\hline Method & Survey items & & Measuring time \\
\hline \multirow{2}{*}{$\begin{array}{c}\text { Field } \\
\text { measurement }\end{array}$} & $\begin{array}{l}\text { Different end-use loads of all } \\
\text { kinds of fuel }\end{array}$ & $\begin{array}{l}\text { Electricity } \\
\text { Gas } \\
\text { Kerosene }\end{array}$ & $\begin{array}{c}\text { Measured every minute } \\
\text { Measured every } 5 \text { minutes } \\
\text { Measured every } 5 \text { minutes }\end{array}$ \\
\hline & \multicolumn{2}{|c|}{$\begin{array}{l}\text { Indoor air temperature } \\
\text { (1.1m above floor) }\end{array}$} & Measured every 15 minutes \\
\hline $\begin{array}{l}\text { Questionnaire } \\
\text { survey }\end{array}$ & \multicolumn{2}{|c|}{$\begin{array}{l}\text { Lifestyle, Utilization of equipment, Annual } \\
\text { income, etc. }\end{array}$} & Once only \\
\hline Inquiring survey & Other issues, such as basic buil & g information & Once only \\
\hline
\end{tabular}

Fig.2. Measuring instruments (from left to right: electricity, gas, kerosene and air temperature)

The building energy consumption was broken down into eight major end-use loads: 1) HVAC, 2) hot water supply (HWS), 3) kitchen (KITC, including cooking and other 
kitchen equipment such as dishwasher and range hood), 4) lighting (LIGHT), 5) refrigerator (REF), 6) amusement and information (A\&I, such as television, telephone, and computer, etc.), 7) housework and sanitary (HOUSE, such as washing machine, vacuum, and electrical shaver, etc.), and 8) others (OTHER, unidentified usage such as electrical shutter and all the unclear items).

Scrutinizing the data from the 80 buildings, researchers found that only 67 sets were complete, while 13 had missing values of energy consumption data. Data reduction and aggregation was then performed to obtain a smaller representation of the original data. For example, diverse energy unit of different kinds of primary energy sources used by the various buildings, including electricity, natural gas, and kerosene, was converted to MJ based on conversion coefficients in Table 2 so they could be added directly. Then, readings of each end-use load at different intervals (e.g., 1 or 5 minutes) were averaged over each month. The resulting data was stored in a database.

Table 2: Conversion coefficients of different fuels

\begin{tabular}{ccc}
\hline Fuel & Conversion coefficient & Unit \\
\hline Electricity & 3.6 & $\mathrm{MJ} / \mathrm{kWh}$ \\
City gas (4A-7C) & 20.4 & $\mathrm{MJ} / \mathrm{Nm}^{3}$ \\
City gas (12A-13C) & 45.9 & $\mathrm{MJ} / \mathrm{Nm}^{3}$ \\
Liquefied petroleum gas (LPG) & 50.2 & $\mathrm{MJ} / \mathrm{Nm}^{3}$ \\
Kerosene & 36.7 & $\mathrm{MJ} / \mathrm{L}$ \\
\hline
\end{tabular}

\subsection{Selection of typical parameters}

The main parameters that could generally represent the four influencing factors unrelated to the occupant behavior should be identified before the cluster analysis. Based on the characteristics of residential buildings in Japan, twelve representative parameters of the four influencing factors were captured from the database and are outlined in Table 3:

Table 3: Representative parameters of the four influencing factors

\begin{tabular}{cllcc}
\hline Influencing factors & \multicolumn{1}{c}{ Representative parameters } & Category & Unit & Abbreviation \\
\hline \multirow{2}{*}{$\begin{array}{c}\text { City } \\
\text { Climate }\end{array}$} & (i) Annual mean air temperature & numerical & ${ }^{\circ} \mathrm{C}$ & $\mathrm{T}$ \\
& (ii) Annual mean relative humidity & numerical & & RH \\
& (iii) Annual mean wind speed & numerical & $\mathrm{m} / \mathrm{s}$ & WS \\
& (iv) Annual mean global solar & numerical & $\mathrm{MJ} / \mathrm{m}^{2}$ & RA \\
radiation & (i) House types ${ }^{\mathrm{a}^{*}}$ & categorical & & HT \\
Building-related & (ii) Building area & numerical & $\mathrm{m}^{2}$ & BA \\
characteristics & (iii) Equivalent leakage area & numerical & $\mathrm{cm}^{2} / \mathrm{m}^{2}$ & ELA \\
& (iv) Heat loss coefficient & numerical & $\mathrm{W}^{\mathrm{c}^{*}} \mathrm{~m}^{3} \mathrm{~K}$ & HLC \\
\hline
\end{tabular}




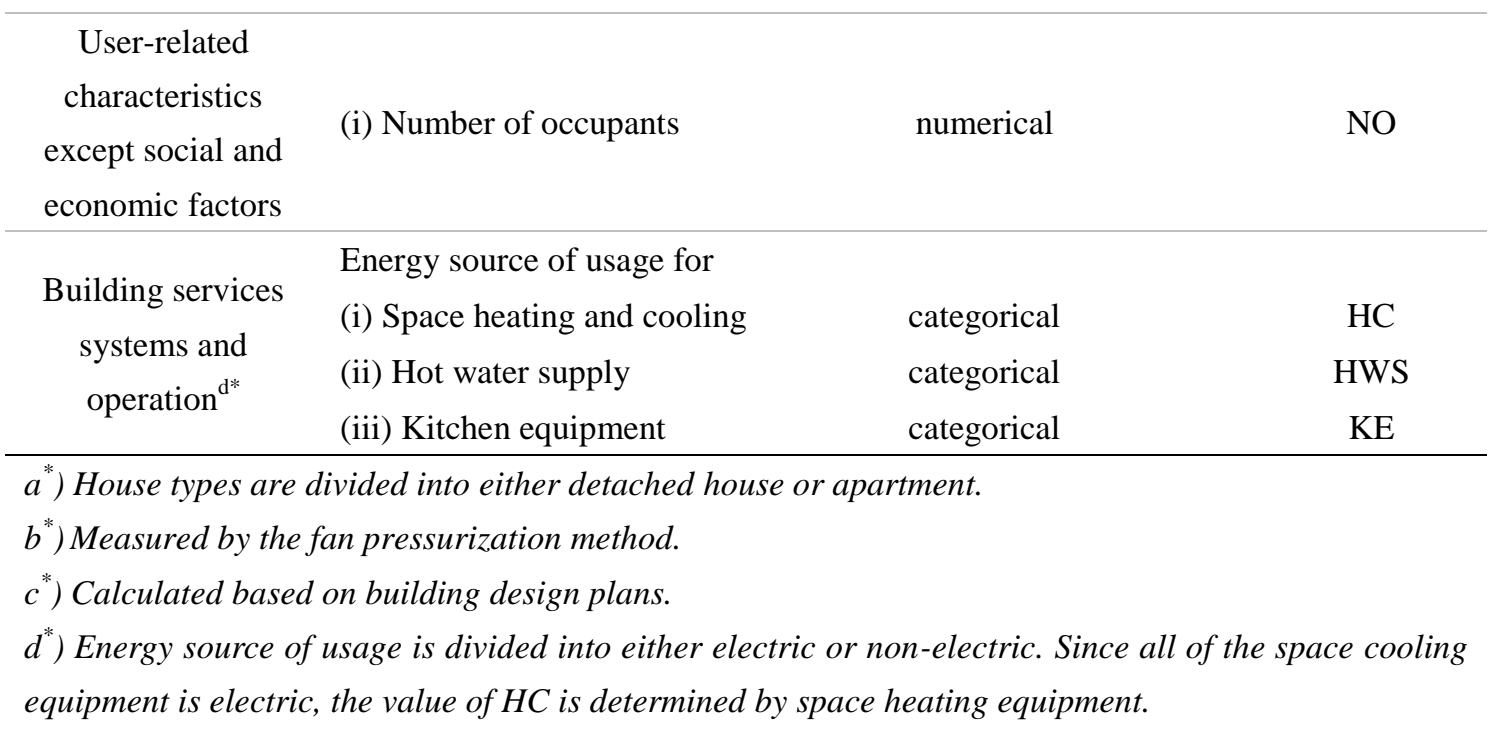

\subsection{Results and discussion}

\subsubsection{Grey relational grades}

The ultimate goal of this study is to identify the influences of the occupant behavior on the building energy consumption. Therefore, annual building energy use intensity (EUI) in 2003 was selected as the objective sequence in GRA, and accordingly, there is no need to consider the building area independently. Among the remaining eleven parameters, four weather parameters are time-series variables that can be viewed as a function of time. In order to take both the impact of season and regional climate difference into consideration, grey relational grades were first calculated for each building based on monthly building EUI and local monthly weather parameters [15]; then, an average was taken over grey relational grades in each district. For the other seven parameters, considering the size of database, grey relational grades were calculated on all the buildings.

The results of GRA are given in Table 4. It can be seen that, with respect to weather parameters, generally outdoor air temperature influenced EUI more significantly than the other three parameters, especially in the cold districts, i.e. Hokkaido and Tohoku. At the same time, the number of occupants and the heat loss coefficient had noticeable impact on the building energy performance, since the grey relational grades of these two parameters are between 0.7 and 0.8 . This implies that these two parameters deserve more attention in the building design phase.

Table 4: Grey relational grades for each district

\begin{tabular}{cccccccccccc}
\hline \multirow{2}{*}{ District } & \multicolumn{10}{c}{ Grey relational grades } \\
\cline { 2 - 13 } & T & WS & RH & RA & NO & HLC & ELA & HT $^{\mathrm{a}^{*}}$ & HC $^{\mathrm{b}^{*}}$ & $\mathrm{HWS}^{\mathrm{b}^{*}}$ & $\mathrm{KE}^{\mathrm{b}^{*}}$ \\
\hline Hokkaido & 0.799 & 0.584 & 0.620 & 0.683 & 0.701 & 0.780 & 0.490 & 0.617 & 0.537 & 0.514 & 0.551 \\
\cline { 3 - 11 }
\end{tabular}




$\begin{array}{ccccc}\text { Tohoku } & 0.831 & 0.555 & 0.765 & 0.662 \\ \text { Hokuriku } & 0.772 & 0.532 & 0.644 & 0.716 \\ \text { Kanto } & 0.737 & 0.601 & 0.732 & 0.641 \\ \text { Kansai } & 0.712 & 0.580 & 0.695 & 0.690 \\ \text { Kyusyu } & 0.654 & 0.605 & 0.661 & 0.675\end{array}$

$a^{*}$ The two states of house types, i.e., detached house and apartment, are transformed to [0, 1].

$b^{*}$ The two states of these three parameters, i.e., electrical and non-electrical, are transformed to [0, 1].

\subsubsection{Cluster analysis}

After data preprocessing and the calculation of the grey relational grades, i.e. weighted coefficients of the selected parameters in Table 3, cluster analysis was conducted using the open-source data mining software WEKA. The results of cluster analysis are given in Table 5. With the consideration of the size of the database, four clusters were determined by the K-means algorithms based on Euclidean distance measures. Cluster centroids, which represent the mean value for each dimension, were used to characterize the clusters. For example, it can be seen that cluster 1, in comparison with the other clusters, is a segment of buildings representing a high outdoor air temperature (the cluster centroid of $\mathrm{T}$ in this cluster is 0.609 , which is higher than that in the other three clusters), detached houses (the cluster centroid of HT in this cluster is 0 , indicating that all the buildings in this cluster are detached house), high heat loss coefficients, low equivalent leakage areas, small number of occupants, non-electrical hot water supplies and kitchen equipment, etc. Similarly, the other clusters can be explained as follows: cluster 2 can be mainly characterized as high solar radiation, large number of occupants, electrical space heating and cooling, and electrical kitchen equipment. Cluster 3 is a segment of buildings representing a low outdoor air temperature, low heat loss coefficients, high equivalent leakage area, and non-electrical hot water supplies. Cluster 4 can be mainly characterized as high outdoor relative humidity, non-electrical space heating and cooling, and electrical kitchen equipment. In addition, the centroid of all the data is also given for comparison with the cluster centroids, as shown in Full Data column in Table 5. The internal cohesion and external separation for the clusters based upon the eleven attributes imply that these attributes have the most similar holistic effects on the building energy performance in the same cluster, while the effects are significantly distinct for the buildings in different clusters.

Table 5

Centroid of each cluster and statistics on the number and percentage of instances assigned to different clusters

\begin{tabular}{cccccc}
\hline \multirow{2}{*}{ Attribute } & \multirow{2}{*}{ Full Data } & \multicolumn{5}{c}{ Cluster } \\
\cline { 3 - 6 } & & 1 & 2 & 3 & 4 \\
\hline T & 0.451 & 0.609 & 0.483 & 0.312 & 0.408 \\
WS & 0.313 & 0.316 & 0.303 & 0.339 & 0.302 \\
RH & 0.395 & 0.262 & 0.417 & 0.428 & 0.439 \\
RA & 0.347 & 0.318 & 0.370 & 0.343 & 0.343
\end{tabular}




\begin{tabular}{cccccc} 
HT & 0.166 & 0.000 & 0.134 & 0.411 & 0.116 \\
HLC & 0.183 & 0.254 & 0.154 & 0.116 & 0.229 \\
ELA & 0.394 & 0.291 & 0.413 & 0.460 & 0.390 \\
NO & 0.275 & 0.216 & 0.320 & 0.234 & 0.296 \\
HC & 0.305 & 0.331 & 0.000 & 0.501 & 0.537 \\
HWS & 0.307 & 0.514 & 0.067 & 0.514 & 0.289 \\
KE & 0.222 & 0.551 & 0.000 & 0.514 & 0.000 \\
Clustered instances and proportion & $67(100 \%)$ & $13(19 \%)$ & $23(34 \%)$ & $15(22 \%)$ & $16(24 \%)$ \\
\hline
\end{tabular}

\subsubsection{Effects of occupant behavior}

\subsubsection{End-use load shapes}

After the generation of four clusters, different end-use loads of various buildings in each cluster were averaged over one year. Fig. 3 shows the average annual EUI of different end-use loads for each cluster. The proportion of each end-use load to the whole is also given above the corresponding bar.

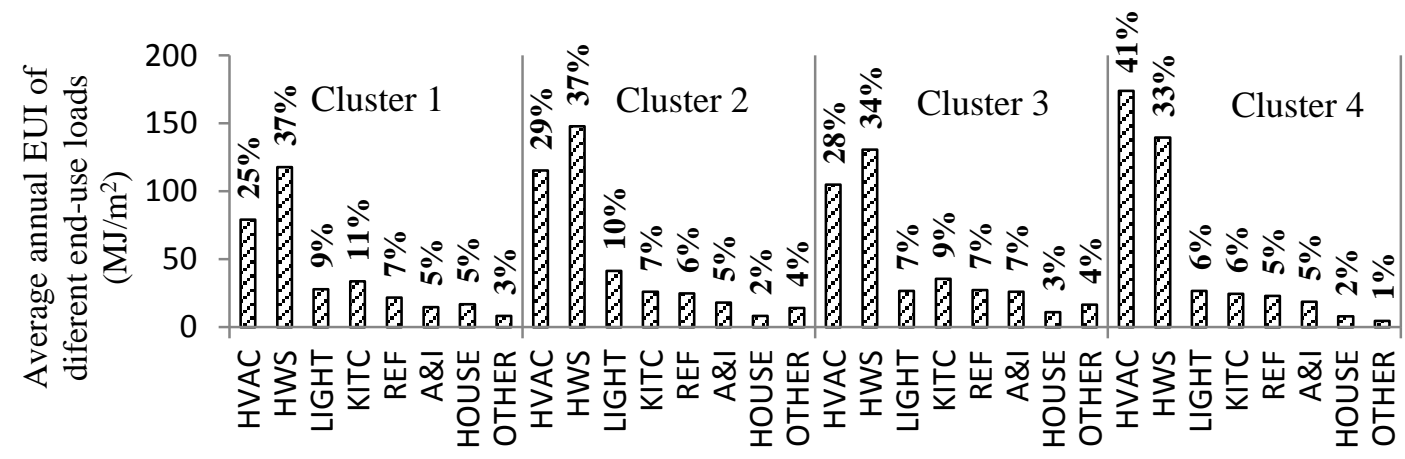

End-use loads

Fig. 3. Average annual EUI of different end-use loads

As shown in Fig. 3, hot water supply and HVAC form the two largest categories of end-use loads in terms of average annual EUI in all four clusters, while housework and sanitary and 'others' have a modest contribution. Also, the two largest loads far exceed the other six end-use loads that do not have significant variations in the proportion among most of the clusters. This indicates that occupants in different clusters had similar behavior. Moreover, the proportions of both hot water supply and HVAC remain approximately steady among these clusters, except that there is a noticeable increase in the HVAC proportion in Cluster 4, which is mainly characterized by medium-low outdoor air temperature and non-electrical space heating equipment. This increase may be partly caused by two factors: 1) the high electricity rate in Japan, and 2) the high efficiency of non-electrical space heating devices such as kerosene space heaters. A high electricity rate tends to restrict occupants' usage of electrical heating/cooling equipment in the other three clusters, while high efficiency of non-electrical space heating devices encourages occupants' utilization of them in Cluster 4, thereby increasing energy consumption. Therefore, a rational combination of electricity rates and primary heating/cooling sources could help reduce building energy consumption through influencing occupant behavior. 


\subsubsection{Variability in annual EUI of different end-use loads induced by occupant}

behavior

In order to examine the variability in annual EUI of different end-use loads that is caused by the occupant behavior, the end-use loads in each cluster were normalized and plotted. Fig. 4 depicts a box plot of normalized annual EUI of different end-use loads. The annual EUI of each building is normalized by the mean value of all the buildings in that cluster, thus highlighting the variability and allowing all the end-use loads to be plotted together on the same scale. As shown in Fig. 4, a large variability that ranges from close to zero to about four times upon the mean value is induced by the user behavior. Since the end-use loads in each building is normalized by the mean value of all the buildings in that cluster, the value of end-use loads ranges from zero to twice as many as the mean value was considered to be an insignificant variation. Accordingly, the threshold value for significant variation is defined as 2 (illustrated by the dash line). Except for HWS and REF, the range of the other six end-use loads exceeds the threshold value in most of the clusters. Such high variability implies that there still remains great potential for energy saving by improving occupant behavior related to these six domestic end-use loads. Contrarily, considering the relatively narrow range of HWS and REF, there could be little expectation of reducing energy consumption in these areas via improving occupant behavior.

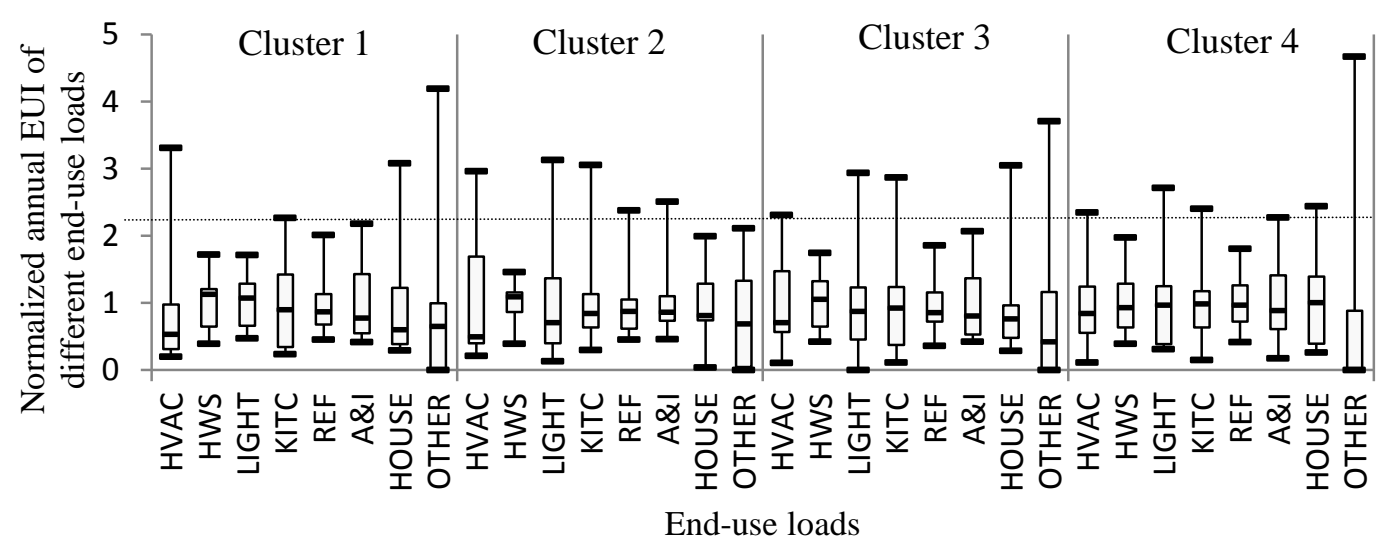

Fig. 4. Boxplot of normalized annual EUI of different end-use loads 


\subsubsection{Reference building and energy-saving potential}

In order to evaluate energy-saving potential for the four clusters, the reference building for each cluster was first defined. The characterization of the reference building was carried out by identifying the building with the energy consumption closest to the cluster energy consumption centroid in terms of Euclidean distance and end-use loads. The annual EUI of different end-use loads of a reference building for each cluster is given in Table 6.

Table 6

Annual EUI of different end-use loads of reference building for each cluster $\left(\mathrm{MJ} / \mathrm{m}^{2}\right)$

\begin{tabular}{cccccccccc}
\hline & HVAC & HWS & LIGHT & KITC & REF & A\&I & HOUSE & OTHER & SUM \\
\hline Cluster 1 & 77 & 165 & 31 & 24 & 25 & 12 & 29 & 0 & 363 \\
Cluster 2 & 45 & 161 & 39 & 25 & 22 & 20 & 7 & 12 & 332 \\
Cluster 3 & 154 & 141 & 33 & 42 & 20 & 13 & 6 & 0 & 409 \\
Cluster 4 & 188 & 212 & 34 & 25 & 15 & 19 & 11 & 0 & 504 \\
\hline
\end{tabular}

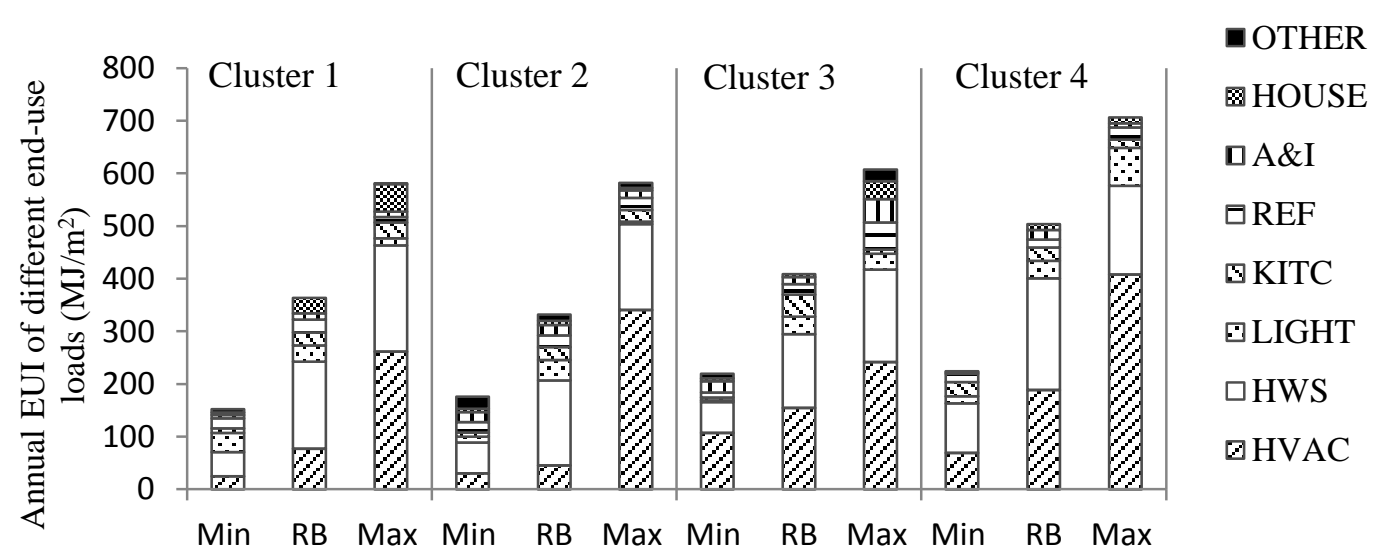

Reference building and buildings with the minimum and maximum EUI

Fig. 5. Stacked-column diagram of annual EUI of different end-use loads of three typical buildings

Fig. 5 shows the stacked-column diagram of annual EUI of different end-use loads of three typical buildings in the four clusters: a reference building (RB) and buildings with the minimum (Min) and maximum (Max) annual EUI. Occupant behavior led to a huge difference between these three different buildings in each cluster. In this study, annual EUI of different end-use loads of a reference building was taken as a baseline. Accordingly, the energy-saving potential of a building with a larger annual EUI than that of a reference building could be determined by computing the difference between them. For example, the potential energy savings that could be achieved by improving occupant behavior for the buildings with the maximum annual EUI in the four clusters, i.e. $\mathrm{EUI}_{\mathrm{Max}}-\mathrm{EUI}_{\mathrm{RB}}$, were $281 \mathrm{MJ} / \mathrm{m}^{2}, 250 \mathrm{MJ} / \mathrm{m}^{2}, 198 \mathrm{MJ} / \mathrm{m}^{2}$, and $202 \mathrm{MJ} / \mathrm{m}^{2}$, respectively. Moreover, comparison with a reference building provided a means of examining which end-use load seemed to have the greatest potential for energy conservation. For instance, comparison between the building with the maximum 
annual EUI and the reference building in each cluster indicated that HVAC contributed the most towards energy saving, while HWS had a negligible contribution. This result is consistent with the conclusion drawn from Fig. 3. Similarly, other end-uses loads with noticeable energy-saving potential in each cluster could be identified, such as housework and sanitary in Cluster 1 and lighting in Cluster 4. Such information can help building owners realize that which occupant behavior should be modified in practice to effectively improve building energy performance. Further, based on this information, a better effect may be achieved if building occupants receive an energy-saving education and tips on how to improve their behavior. It should be noted that, in comparison with a reference building, buildings with the minimum annual EUI in the four clusters not only had lower HVAC EUI, but also had much smaller HWS EUI. A possible explanation for this is that occupants in these buildings reduced energy consumption by being concerned about the cost in living standards. For example, these occupants may decrease the frequency of utilization of room air conditioners in the cooling season, even though the indoor temperature is not the best comfort temperature. Further field investigation is needed to identify the real reasons.

\subsubsection{Monthly variations of end-use loads induced by occupant behavior}

In order to examine the effects of occupant behavior on end-use loads over time and buildings, monthly variations of average end-use loads in each cluster were plotted in semi-logarithmical graphs, as shown in Fig. 6 to 9. Clearly HVAC shows a significant variation in all the four clusters. Generally, the peak of HVAC occurred in the heating season, especially in December and January, while the trough of HVAC occurred in the cooling season, especially June and July. This may have occurred because four districts (i.e., Hokuriku, Kanto, Kansai, and Kyushu) have a moderate climate and the other two (Hokkaido, Tohoku) are located in a cold climate, and cooling energy demand is considerably lower than heating energy demand. At the same time, HVAC in Cluster 3, characterized by the lowest outdoor air temperature, had the biggest peak-to-trough ratio. This indicates that weather conditions significantly influenced occupant behavior, thereby impacting building energy consumption. With respect to HWS, its variation is noticeable, considering the absolute magnitude of the variation is comparatively large. In general, the peak of HWS occurred in December or January, while the trough occurred in August or September. Evidently this was also caused by weather conditions, especially outdoor air temperature. With regard to LIGHT, KITC, REF, and A\&I, these four curves bear a remarkable similarity to each other in the four clusters, and almost all of them vary by less than $20 \%$ from the mean. This indicates that these households tended to maintain their lifestyles, and the level of their general indoor activities associated with these end-use loads did not fluctuate wildly from month to month. In addition, the remaining two smaller end-use loads, i.e., HOUSE and OTHER, showed a marked seasonal variation in the four clusters, while the absolute magnitude of the variation is comparatively small. Basically the end-use loads in a heating season are higher than in a cooling season. A further investigation of corresponding occupant-behavior patterns needs to be performed to explain the 
reasons for this variation.

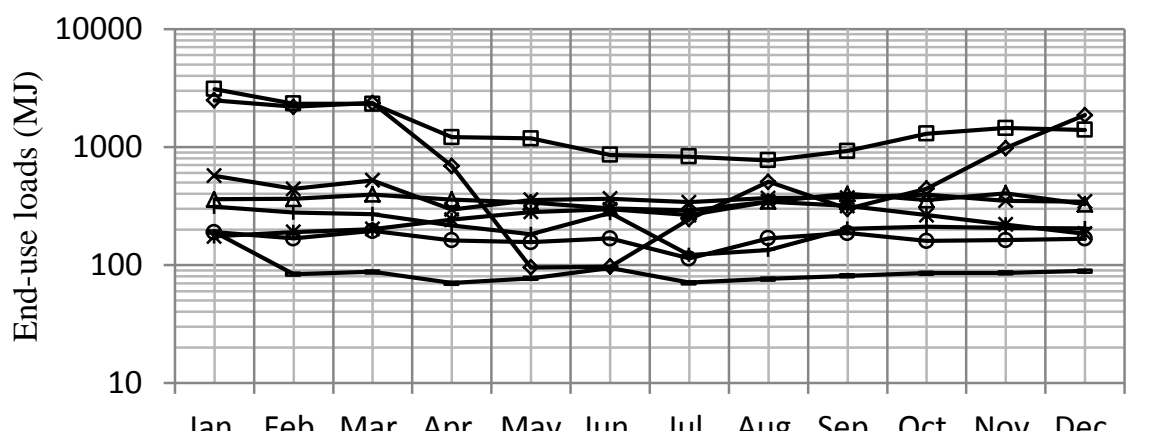

$\multimap$ HVAC

๑-HWS

$\rightarrow$ LIGHT

* KITC

$\rightarrow$ * REF

$\rightarrow$ A\&I

Jan. Feb. Mar. Apr. May Jun. Jul. Aug. Sep. Oct. Nov. Dec.

— HOUSE

Month OTHER

Fig. 6. Monthly variation of end-use loads in Cluster 1

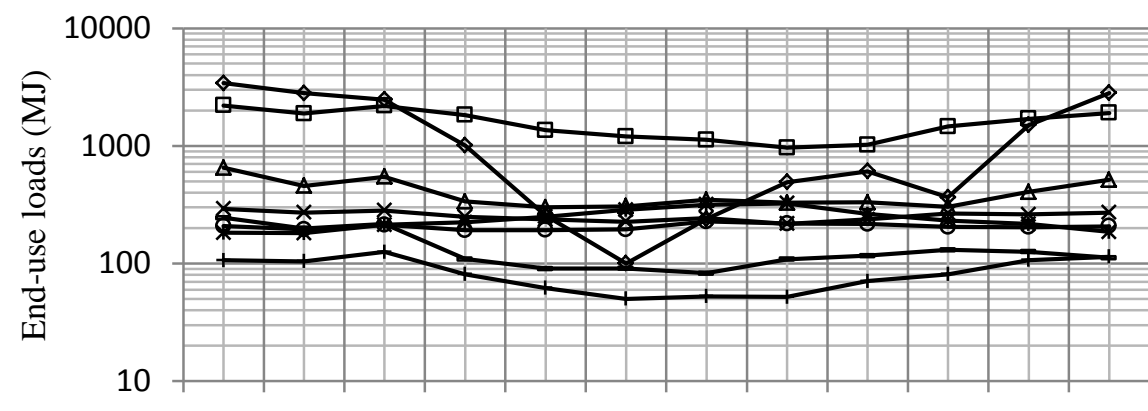

$\multimap$ HVAC

$\rightarrow-H W S$

$\rightarrow$ LIGHT

$*$ KITC

$\rightarrow$ REF

$\rightarrow A \&$

Jan. Feb. Mar. Apr. May Jun. Jul. Aug. Sep. Oct. Nov. Dec.

$\longrightarrow$ HOUSE

Month OTHER

Fig. 7. Monthly variation of end-use loads in Cluster 2

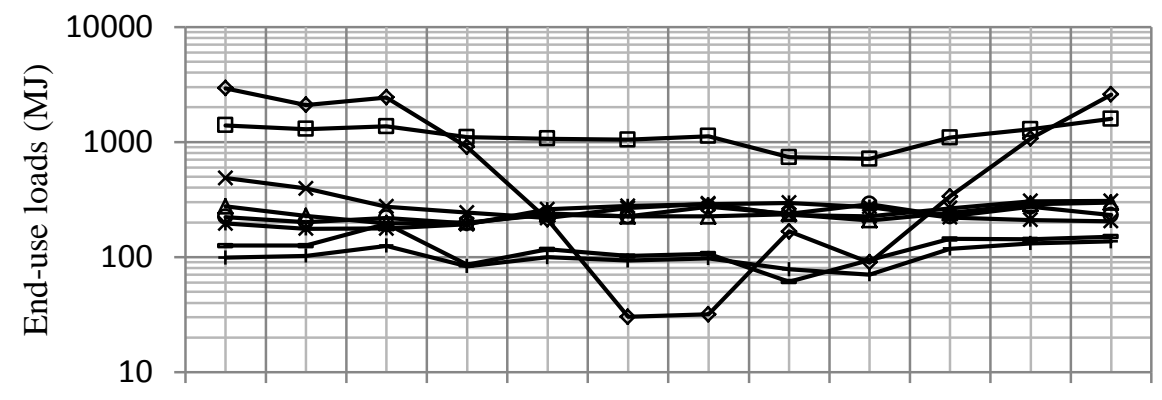

$\multimap$ HVAC

๑-HWS

$\rightarrow$ LIGHT

$\rightarrow$ KITC

* REF

Jan. Feb. Mar. Apr. May Jun. Jul. Aug. Sep. Oct. Nov. Dec.

$\rightarrow A \&$

Month

Fig. 8. Monthly variation of end-use loads in Cluster 3 


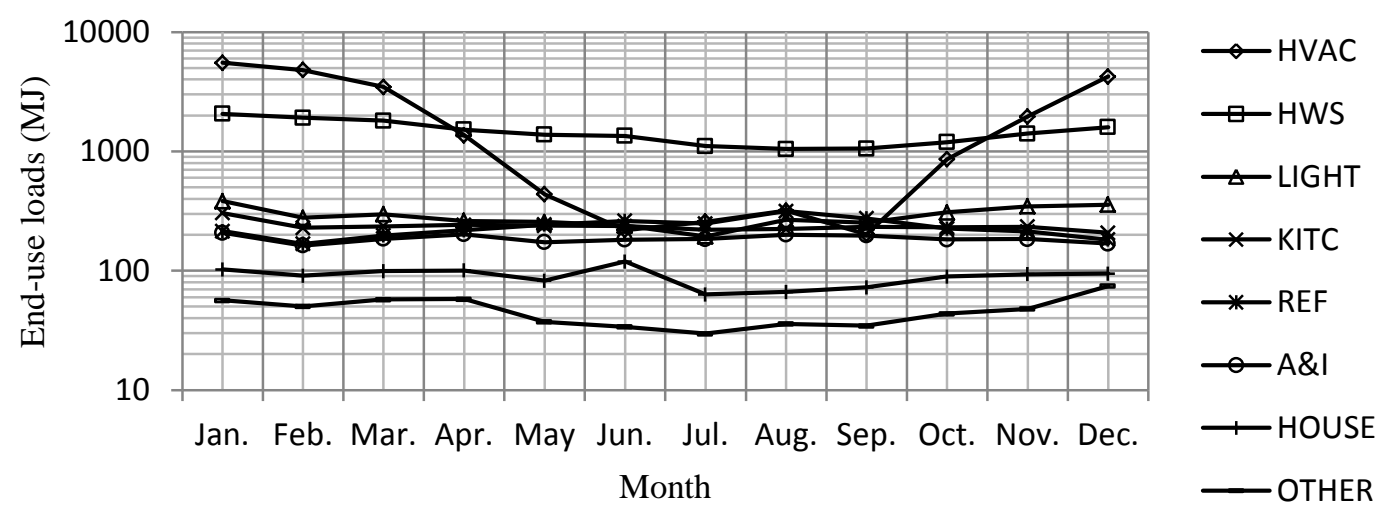

Fig. 9. Monthly variation of end-use loads in Cluster 4

\subsubsection{Monthly average indoor temperature of air-conditioned room}

Different occupant behavior, especially those associated with HVAC, can significantly affect indoor climate, which in turn will have an influence on occupant behavior, thereby causing dramatic differences in building energy consumption. Therefore, the effects of occupant behavior on building energy consumption should be understood and interpreted in conjunction with the investigation of indoor climate. Figures 10-13 show the monthly average living-room temperature of three typical buildings in each cluster: the reference building (RB) and buildings with the maximum and minimum annual EUI (Max and Min). These selected living rooms had air conditioners and/or heating equipment. As shown in Fig. 10, there is a significant difference between living-room temperatures of the three buildings in the cooling season and a minor difference in other seasons. The living room of Max was maintained at a temperature of about $24{ }^{\circ} \mathrm{C}$ in the cooling season. At the same time, the room temperature of Min was around $5{ }^{\circ} \mathrm{C}$ higher than that of Max, and the room temperature of $\mathrm{RB}$ was generally between that of Max and Min in this season. Considering that Cluster 1 is characterized by the highest outdoor air temperature, it can be deduced that the frequency of utilization of room air conditioners in the cooling season in these three buildings can be ranked as: Max > RB > Min. With respect to the other three clusters, Fig. 11-13 shows that the living room of Max was maintained at a temperature of about $24{ }^{\circ} \mathrm{C}$ all year, while living-room temperatures of $\mathrm{RH}$ and Min varied with the outdoor air temperature. Clearly the frequency of utilization of space cooling/heating equipment in the three buildings in these three clusters has the same order as that in Cluster 1 in both heating and cooling seasons. These results suggest that occupant behavior that seeks thermal comfort normally results in high energy consumption. Therefore, there has to be a trade-off between human thermal comfort and building energy consumption, and it is necessary to strike a balance between achieving a high comfort level and reducing energy consumption through modifying occupant behavior. 


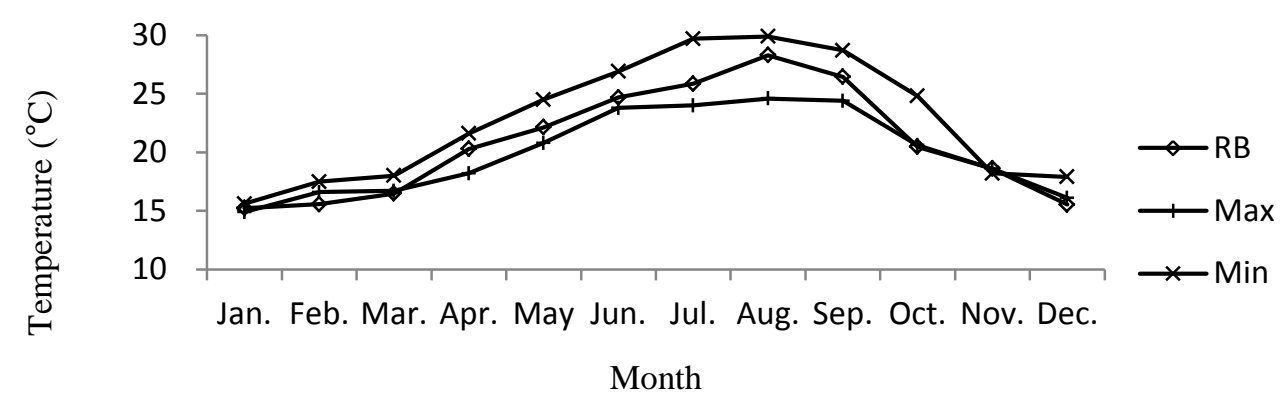

Fig. 10. Monthly average living-room temperature of three typical buildings in Cluster 1

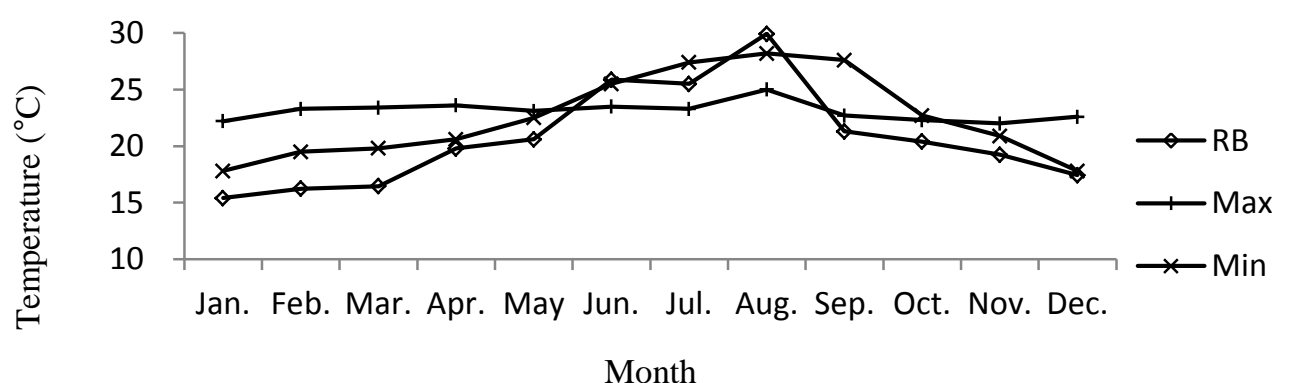

Fig. 11. Monthly average living-room temperature of three typical buildings in Cluster 2

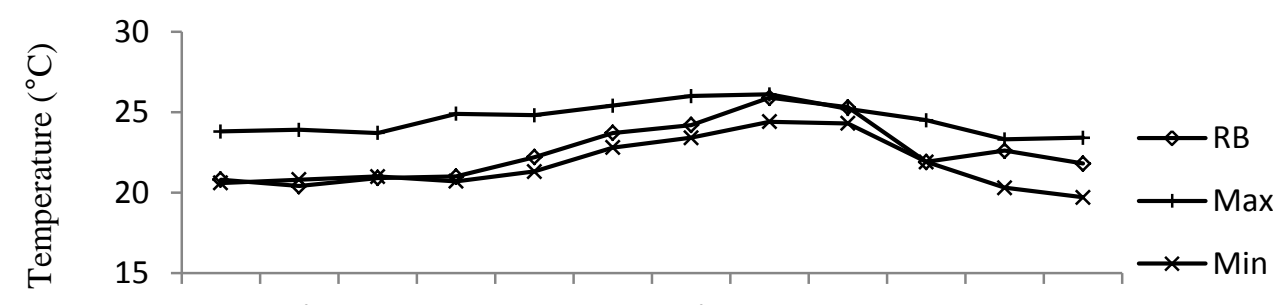

Jan. Feb. Mar. Apr. May Jun. Jul. Aug. Sep. Oct. Nov. Dec.

Month

Fig. 12. Monthly average living-room temperature of three typical buildings in Cluster 3

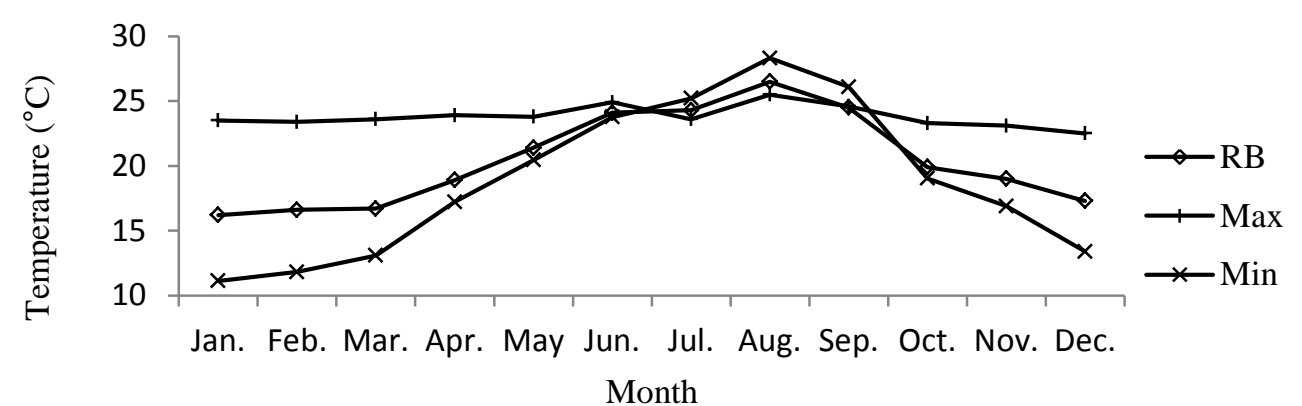

Fig. 13. Monthly average living-room temperature of three typical buildings in Cluster 4

\section{Summary and Conclusions}

The main purpose of this paper includes the development of a novel data analysis methodology through clustering techniques for identifying the effects of occupant behavior on building energy consumption. It is realized by organizing similar buildings among all the investigated buildings into various groups based on the four influencing factors unrelated to user behavior, so that for each building in the same group the four factors have similar full effects on energy consumption. Min-max 
normalization techniques are performed as a data preprocessing step to deal with the inconsistencies of different attributes. Grey relational analysis is also carried out, and grey relational grades, a measure of relevancy between two factors, are used as weighted coefficients of attributes in cluster analysis.

In order to demonstrate its applicability, this methodology was applied to a group of residential buildings located in six different districts of Japan. Energy-related data of these buildings was measured, and a database was developed after scrutinizing the measured data. Twelve attributes were captured from the database to represent the influencing factors unrelated to occupant behavior. K-means method was selected in cluster analysis and four clusters were obtained as a result.

In these four clusters the effects of occupant behavior on building energy consumption were examined at the end-use level. End-use variations over time and buildings induced by occupant behavior were analyzed. Also, as a preliminary step toward identifying energy-saving potential, a reference building was defined as the building whose energy consumption was the closest to cluster energy consumption centroid in terms of Euclidean distance and end-use loads. Moreover, indoor climate was investigated to better understand and interpret the effects of occupant behavior.

This proposed method allows researchers to evaluate building energy-saving potential by improving user behavior, and provides multifaceted insights into building energy end-use patterns associated with occupant behavior. The results obtained could help prioritize efforts of modification of occupant behavior to reduce building energy consumption, and also could be used to improve modeling of user behavior in numerical simulation.

The main focus of future research should be placed on identifying appropriate building sample sizes and number of clusters, selecting typical attributes that can adequately represent the influencing factors unrelated to occupant behavior, since these measures will provide more precise effects of occupant behavior. In addition, more case studies in different sectors, such as commercial buildings and office buildings, should be conducted to further improve building energy performance and policy formulation.

\section{Acknowledgements}

The authors would like to express their gratitude to the Public Works and Government Services Canada, and Concordia University for the financial support.

\section{References}

[1] C. F. Reinhart, Lightswitch-2002: a model for manual and automated control of electric lighting and blinds, Solar Energy 77 (1) (2004), pp. 15-28.

[2] D. Bourgeois. Detailed occupancy prediction, occupancy-sensing control and advanced behavioral modeling within whole-building energy simulation. Ph.D. thesis, l'Universite Laval, 
Quebec, 2005.

[3] H. B. Rijal, P. Tuohy, M. A. Humphreys, J. F. Nicol, A. Samuel, J. Clarke, Using results from field surveys to predict the effect of open windows on thermal comfort and energy use in buildings, Energy and Buildings 39 (7) (2007), pp. 823-836.

[4] P. Hoes, J. L. M. Hensen, M. G. L. C. Loomans, B. de Vries, D. Bourgeois, User behavior in whole building simulation, Energy and Buildings 41(3) (2009), pp. 295-302.

[5] H. Nakagami, Lifestyle change and energy use in Japan: Household equipment and energy consumption, Energy 21(12) (1996), pp. 1157-1167.

[6] L. Lopes, S. Hokoi, H. Miura, K. Shuhei, Energy efficiency and energy savings in Japanese residential buildings - research methodology and surveyed results, Energy and Buildings 37(7) (2005), pp. 698-706.

[7] Z. Yu, F. Haghighat, B. C. M. Fung, H. Yoshino, A decision tree method for building energy demand modeling, Energy and Buildings 42(10) (2010), pp. 1637-1646.

[8] A. F. Emery, C. J. Kippenhan, A long-term study of residential home heating consumption and the effect of occupant behavior on homes in the Pacific Northwest constructed according to improved thermal standards, Energy 31(5) (2006), pp. 677-693.

[9] J. Ouyang, K. Hokao, Energy-saving potential by improving occupants' behavior in urban residential sector in Hangzhou City, China, Energy and Buildings 41(7) (2009), pp. 711-720.

[10] J. Han, M. Kamber, Data mining concepts and techniques, Elsevier Inc., San Francisco (2006).

[11] WEKA, The University of Waikato. Software, http://www.cs.waikato.ac.nz/ml/weka/

[12] J. Deng, Introduction to grey system, Journal of Grey System 1 (1989), pp. 1-24.

[13] C. Fu, J. Zheng, J. Zhao, W. Xu, Application of grey relational analysis for corrosion failure of oil tubes, Corrosion Science 43(5) (2001), pp. 881-889.

[14] http://www.data.jma.go.jp/obd/stats/data/en/smp/index.html

[15] Climate Statistics, Japan Meteorological Agency. Monthly Mean and Monthly Total Tables, http://www.data.jma.go.jp/obd/stats/data/en/smp/index.html 